\title{
Absence of R140Q mutation of isocitrate dehydrogenase 2 in gliomas and breast cancers
}

\author{
STÉPHANE RAYNAUD ${ }^{1 *}$, NADINE CARBUCCIA ${ }^{1 *}$, CAROLE COLIN $^{2}$, JOSÉ ADÉLAIIDE $^{1}$, \\ MARIE-JOELLE MOZZICONACCI ${ }^{1}$, PHILIPPE METELLUS ${ }^{3}$, OLIVIER CHINOT ${ }^{4}$, DANIEL BIRNBAUM ${ }^{1}$, \\ MAX CHAFFANET $^{1}$ and DOMINIQUE FIGARELLA-BRANGER ${ }^{2}$ \\ ${ }^{1}$ Centre de Recherche en Cancérologie de Marseille, Laboratoire d' Oncologie Moléculaire, UMR891 Inserm, \\ Institut Paoli-Calmettes, 13009 Marseille; ${ }^{2}$ INSERM U911, Centre de Recherche en Oncologie et \\ Oncopharmacologie (CRO2), Angiogenèse, Invasivité et Microenvironnement Tumoral; \\ Services de ${ }^{3}$ Neurochirurgie et ${ }^{4}$ Neuro-oncologie, AP-HM, 13005 Marseille, France
}

Received May 11, 2010; Accepted June 23, 2010

DOI: 10.3892/ol_00000156

\begin{abstract}
Somatic mutations of isocitrate dehydrogenase (IDH)-1 and IDH2 proteins have been described in gliomas. The mutations target the R132 amino acid residue and the R172 residue in IDH1 and IDH2, respectively. The same mutations were observed in acute myeloid leukemias with normal karyotype, but a new mutation in IDH2 (R140Q substitution) was detected in malignant myeloid diseases and appears to be the most frequent IDH mutation in these pathologies. To the best of our knowledge, no study thus far has reported the presence of this R140Q mutation in IDH2 in tumors of the nervous system and breast cancers. We evaluated $I D H 1$ and $I D H 2$ exon 4 in 48 low-grade gliomas, 58 primary glioblastomas and 94 breast cancers to evaluate the frequency of mutation and investigated the R140Q substitution in IDH2. The results were compared to our recently obtained results in hematopoietic diseases. The frequency of IDH1 and IDH2 mutations in our panel of gliomas was similar to previously reported mutations. No IDH2 R140 mutation was observed. Compared to hematopoietic diseases, the IDH2 R172 mutation was also more rare and IDH1 mutations more prominent in tumors of the nervous system. No IDH1 or IDH2 mutation was detected in the 94 breast cancer samples. Thus, the IDH2 R140 mutation appears to be restricted to hematopoietic diseases.
\end{abstract}

\footnotetext{
Correspondence to: Dr Max Chaffanet, Centre de Recherche en Cancérologie de Marseille, Laboratoire d' Oncologie Moléculaire, UMR891 Inserm, Institut Paoli-Calmettes, 27 Bd. Leï Roure, 13009 Marseille, France

E-mail: chaffanetm@marseille.fnclcc.fr

${ }^{*}$ Contributed equally
}

Key words: isocitrate dehydrogenase 2, mutations, gliomas, breast cancers, acute myeloid leukemias

\section{Introduction}

Somatic mutations of isocitrate dehydrogenase (IDH)-1 and IDH2 proteins have been described in gliomas. The mutations target the R132 amino acid residue in IDH1 and the R172 residue in IDH2, both of which are encoded by the fourth exon of the corresponding gene (1). Mutations of IDH1 are frequent $(>80 \%)$ in astrocytomas and oligodendrogliomas, whereas they are very rare in primary glioblastomas and other tumors of the nervous system $(2,3)$. IDH2 mutations are rare, but more common in oligodendroglial tumors, as compared to astrocytomas. These mutations are also mutually exclusive with those in IDH1. IDH mutations occur at the substrate binding site and inactivate the standard enzymatic activity (4).

Recently, the same mutations, which are considered to be rare in the majority of common tumors outside gliomas $(5,6)$, were discovered in acute myeloid leukemias (AMLs) with normal karyotype $(7,8)$. In malignant myeloid diseases, a new mutation affecting R140 in IDH2 was detected $(9,10)$. Authors of this study (unpublished data), as well as other investigators $(8,9)$, determined that the R140Q substitution was the most frequent IDH mutation in these pathologies. Thus, mutations of IDH1 and IDH2 were found in $28 \%$ of primary AMLs.

\section{Materials and methods}

Samples. Exons 4 of $I D H I$ and $I D H 2$ were analyzed on panels of DNAs from randomly chosen brain and breast cancer samples. Their sizes were approximately the same as our set of AMLs previously reported (unpublished data). For tumors of the nervous system, the set comprised 48 low-grade gliomas (5 astrocytomas, 22 oligoastrocytomas and 21 oligodendrogliomas) and 58 primary glioblastomas. The breast cancer panel comprised 94 samples, including 60 invasive ductal carcinomas, 7 invasive lobular carcinomas, 4 medullary carcinomas, 5 mixed type, 1 tubular carcinoma, 3 others and 14 not informed.

$D N A$ sequencing. DNA sequencing of IDH1, IDH2 exons 4 was performed. Briefly, purified PCR products $(1 \mu \mathrm{l})$ were used 
Table I. Mutations of IDH1 and IDH2 in four types of human malignant diseases.

Cases $\begin{gathered}\text { No. of IDH1 R132 IDH2 R172 IDH2 R140 } \\ \text { patients }\end{gathered}$

\begin{tabular}{lcccc}
\hline $\begin{array}{l}\text { Low-grade } \\
\text { gliomas }\end{array}$ & 48 & 31 & 1 & 0 \\
$\begin{array}{l}\text { Glioblastomas } \\
\begin{array}{l}\text { Acute myeloid } \\
\text { leukemias }\end{array}\end{array}$ & 64 & 2 & 0 & 0 \\
Breast cancers $^{\mathrm{a}}$ & 94 & 0 & 4 & 11 \\
\hline
\end{tabular}

aOur data on acute myeloid leukemias are shown for comparison.

for sequencing using the Big Dye terminator v1.1 kit (Applied Biosystems), including the forward or reverse primer. After G50 purification, sequences were loaded on an ABI 3130XL automat (Applied Biosystems). The sequence data files were analyzed using SeqScape and Phred/Phrap/Consed softwares. Mutations were confirmed on an independent PCR product.

\section{Results}

We investigated whether the IDH2 R140 new mutation was present in tumors of the nervous system and may have been overlooked. We also aimed to determine whether breast cancers exhibited any IDH2 mutation. We determined the sequence of exon 4 of $I D H I$ and $I D H 2$ genes from the DNA of brain and breast tumor samples.

In low-grade gliomas, we found 31 IDH1 mutations ( 65\%) affecting residue 132 and distributed as follows: $28 \mathrm{R} 132 \mathrm{H}, 2$ R132C and 1 R132S. IDH2 was mutated in only 1 case (not mutated for IDH1) at residue 172 (R172M). In primary glioblastomas, only 2 IDH1 heterozygous mutations (R132H) were noted, but no IDH2 mutation. No IDH1 or IDH2 mutations were found in the 94 breast cancer samples.

\section{Discussion}

The frequency of IDH1 and IDH2 mutations in our panel of gliomas is similar to what has previously been reported $(2,3)$. Despite this high frequency, the IDH2 R140 residue was not affected in our series of neural tumors. Compared to hematopoietic diseases, the IDH2 R172 mutation was also more rare and the IDH1 mutations more prominent in tumors of the nervous system (Table I). IDH mutations were completely absent in breast tumors, as previously noted $(5,6)$. However, no previous study investigated the IDH2 mutation in breast cancer. Our study showed that this disease is not associated with the IDH2 mutation. Thus, the IDH2 R140 mutation appears to currently be restricted to hematopoietic diseases. All IDH arginine mutants produce 2-hydroxyglutarate $(8,10)$. The specific role of the R140 mutation in hematopoietic diseases remains to be established, but may be linked to subtle enzymatic differences between allele mutants.

\section{Acknowledgements}

This study was supported by Inserm, Institut Paoli-Calmettes and by institutional grants to $\mathrm{CRO} 2$. Frozen samples were provided by the AP-HM TumorBank (Authorization no. 2008-70).

\section{References}

1. Yan H, Parsons DW, Jin G, et al: IDH1 and IDH2 mutations in gliomas. N Engl J Med 360: 765-773, 2009.

2. Ohgaki $\mathrm{H}$ and Kleihues P: Genetic alterations and signaling pathways in the evolution of gliomas. Cancer Sci 100: 2235-2241, 2009.

3. Hartmann C, Meyer J, Balss J, et al: Type and frequency of IDH1 and IDH2 mutations are related to astrocytic and oligodendroglial differentiation and age: a study of 1,010 diffuse gliomas. Acta Neuropathol 118: 469-474, 2009.

4. Dong L, White DW, Gross S, et al: Cancer-associated IDH1 mutations produce 2-hydoxyglutarate. Nature 462: 739-744, 2009.

5. Kang MR, Kim MS, Oh JE, et al: Mutational analysis of IDH1 codon 132 in glioblastomas and other common cancers. Int $\mathbf{J}$ Cancer 125: 353-355, 2009.

6. Bleeker FE, Lamba S, Leenstra S, et al: IDH1 mutations at residue p.R132 (IDH1(R132)) occur frequently in high-grade gliomas but not in other solid tumors. Hum Mutat 30: 7-11, 2009.

7. Mardis ER, Ding L, Dooling DJ, et al: Recurring mutations found by sequencing an acute myeloid leukemia genome. N Engl J Med 361: 1058-1066, 2009.

8. Gross S, Cairns RA, Minden MD, et al: Cancer-associated metabolite 2-hydroxyglutarate accumulates in acute myelogenous leukemia with isocitrate dehydrogenase 1 and 2 mutations. J Exp Med 207: 339-344, 2010.

9. Green A and Beer P: Somatic mutations of IDH1 and IDH2 in the leukemic transformation of myeloproliferative neoplasms. $\mathrm{N}$ Engl J Med 362: 369-370, 2010.

10. Ward PS, Patel J, Wise DR, et al: The common feature of leukemia-associated IDH1 and IDH2 mutations is a neomorphic enzyme activity converting $\alpha$-ketoglutarate to 2 -hydroxyglutarate. Cancer Cell 17: 1-10, 2010. 